\title{
THE IMPOSSIBILITY OF QUINE'S INDETERMINACY THEORY
}

\author{
Duncan MacIntosh, Toronto
}

Quine thinks that the Positivists were wrong to suppose that individual experiences determinately attach to individual expressions. But then, of course, the meaning of a sentence can't be a function of its experiential associations; the association of different expressions with the same experiences can't be used as criterial of their synonymy; the analyticity of a sentence can't involve the experience-given meanings of its components conspiring to make it true in the face of all possible experience; there will be no class of expressions privileged to stand in immediate correlation with truth-adjudicating experiences, directly referring to them, and subsequently serving as the expressions of complexes of which all others are equivalent; nor will it be possible to hold the references of theory-sentences reducible to references of observationally basic expressions.

Quine, as a student of language and theory structures, noticed that no sentence is subject to empirical confrontation except on assumption of the truth of sentences not intuitively observational, and that intuitively non-observational sentences face the tribunal of experience as much as observation-sentences, given the context of assumptions, but that the adjudication of sentences is not in general a procedure involving a direct relation between individual sentences and bits of the observable world. Rather, a given sentence is valued true in an observational circumstance in part as a result of that circumstance, and in part as a result of the tacit prior valuation of indefinitely many other sentences, themselves not in turn individually indexed to specific observations - if the observation bears on the given sentence, it also bears on the ancillary background sentences which set it up for valuation in that circumstance.

With experience bearing only on whole systems of sentences, rather than on individual sentences, Quine despaired of finding a way to conceive each sentence as separately controlled by data - by non-linguistic observational conditions. Perhaps it was this that led him to the belief that even in the face of a totality of possible data, there would be room for theoretical variation in science, and that even given all possible information about a language-user's verbal behaviour in conjunction with observable portions of the environment, there would remain undecidable questions of translation.

In any event, he did pick this pessimistic route, and when we take his theory that observational data underdetermine the truth and meaning of expressions, and combine it with his latent empiricism - the view that only relations between expressions and things observable could constitute the meaning and truth of an expression, classically conceived - we get his indeterminacy theory. Where the meaning or 
truth of a sentence are empirically underdetermined, there is no ultimate fact of the matter about them. Likewise, anything about a sentence traditionally thought dependent upon the sentence's meaning will be indeterminate - its ontological commitments, whether it is analytic or synthetic, whether it is consistent with a given sentence, whether a given sentence is its proper philosophical analysis, and so on.

Now, Michael Dummett and Karl Schick have objected to Quine on the ground that, whatever he may say about meaning being indeterminate, he offers a naturalized model for language in which indeterminacy is nowhere to be found. They both interpret him as holding that there are some expressions in languages and theories which are effectively conditioned in their assentibility or truth to adjudicating experiences - and that all other expressions are conditioned in their turn to selections of these expressions. Schick holds that theory-sentences are "conditioned" to observation-sentences, which are conditioned to the stimulus-environment, while Dummett speaks of observation-sentences being "connected" to theory-sentences, with the stimulus-environment impinging on the observation-sentences.

Schick then observes that at worst, radical translation is demonstrated to be a two-stage process, involving first the translation of observation-sentences by their stimulus-condition, and second, the translation of theory-sentences by the observation-sentence complexes which serve as their assentibility-conditions. He then wonders how sentence-meaning and translation could be indeterminate in this schema it seems every sentence has a traceable stimulus-significance, and that these suffice to individuate meanings. He grants that the sign-design forms of the observation sentences to which theory-sentences are keyed in distinct languages are different, but thinks these differences "divide through" in the translational process. Surely, he argues, Quine doesn't think that sign-design differences make for differences in the intuitive meanings of expressions? But then what could make stimulus-synonymous translational hypotheses seem intuitively different? He might also have asked what determines which translation-hypotheses are thought to be in specific competition as the possible meanings of a given away-language expression if for some reason stimulus-synonymy fails to hold at (say) theoretical levels. And whatever it is, why does it fail to be criterial and constitutive of meaning? What could make expressions which should thus be synonymous, seem to be logically incompatible, if Quine's naturalistic account is correct?

Dummett focuses on the connections between sentences in what he takes to be Quine's model. He argues that if experience impinges on the periphery of a language"web", and its effects are inwardly transmitted by the connection between elements in the web - in the articulated structure of the language - then every expression is determinately controlled by experience, via the structural principles of the language. Inter-sentential connections are effectively logical connections, and sentences expressive of the interanimatory principles of the net would be the logical truths of the language. Everything again appears perfectly determinate. Now, if Quine were to hold that the theory sentences interior to the web somehow constrain or promote the assentibility of the sentences in the web in direct encounter with perceptual stimuli, we are entitled to ask in what sense the outer sentences are conditioned at all to the stimulus-environment, while if the interior sentences are susceptible of independent 
behaviour, we are entitled to ask in what sense peripheral sentences impinge upon them. And what would be left of the idea that the sentence network has a structure; that the model gives explanations of the rules governing the behaviour of its constituents? The "model" is no model at all. Its business is the specification of the principles governing language use, and yet these have all been exceptioned to the point of vacuity. The indeterminacy theory looks to be the very antithesis of a model or theory of language.

These seem like very strong objections indeed, and at the very least they bring out important conditions for the adequacy of any theory of language - conditions which will figure again, shortly. But they also fail in two respects. First, they are somewhat uncharitable to Quine, leaving entirely out of account his doctrine of background-language relativity, a doctrine which can go some way in accommodating the foregoing problems. Second, Dummett and Schick nowhere come to terms with the holistic considerations which a theory of language must address, and which may have induced Quine's indeterministic predilections in the first place.

Quine takes the significance of an expression to be a function of its observable conditions of use, as do Positivists. But he thinks that some of these conditions are linguistic; that there are indefinitely many sentences to which one must be disposed to assent before one will assent to a given sentence in a given perceptual circumstance. Together, the perceptual stimuli and the ancillary assumptions determine the assentibility of the given sentence, and this consists in a partial reply to Dummett, illustrating how there is something to explain every verbal disposition in Quine's model. Further, where a given experience inclines assent to a sentence incompatible with ancillary assumptions, two principles decide the distribution of assent - change as little as possible, while maximally accommodating experience. These principles also afford a redefinition of the observation/theory distinction - the most easily revised sentences being observational, the least revisable being logical or analytic, with the remaining being variously theoretic. The resistance of verbal practice to revision is directly proportionate to its ubiquity in the network, logical rules being very pervasive, direct reports of perceptual experience being relatively isolated.

This appears to be a complete answer to Dummett, and we can also see how some of Quine's more radical theses are introduced — among the conditions upon assent for a given sentence are other sentences in the language, but since they are not determinately subject to control by the experiential conditions external to and common between languages, their significance is not specifiable in a way that could be transferred across languages; that would make sense of the idea that expressions in different languages can say the same things. So, to the extent that an expression has purely linguistic assent-conditions (not in turn susceptible to extra-linguistic control), its sense is relativized to the language of discourse (observational-sentences being the most interlinguistically objective). Now, classically, the relation between a sign and what it signifies is arbitrary; the choice of symbol doesn't determine its material content. Yet here, symbol-choice (selection of a language of discourse) does seem to contribute to sense, while since classically this choice is thought irrelevant to the way the world is, there is no worldly fact of the matter about an expression's truth, except relative to a language. Of course, if meaning and truth are language-relative, so is 
ontology, for ontological commitments are part of meanings. Thus translation imposes the ontology of the home-language on the away-language (a similar story may be told about theories empirically equivalent in the whole). Finally, since every verbal disposition is in large measure adopted on the basis of linguistic pragmatics, any disposition, even one taken to exemplify an analytic relation, is revisable in pursuit of economy and accommodation to the data, while no revision should be trivial, owing to the experiential significance which infuses the whole network in various partial degrees of attenuation.

Well, if Dummett is answered, Schick isn't. Classically, the inscriptions, sounds and gestures which are deployed in linguistic activity don't themselves possess an intrinsic sense, but only a functional one. Yet Quine's theory seems to involve this very thing. In holding that the intuitive sense of an expression derives from its observable conditions of use, and that extra-linguistic observables underdetermine expression-sense, he is forced to make up the slack by effectively endowing sentences (qua observable sign-designs) with intuitive sense, or with the capacity to endow such sense to the sentences which they condition.

It is just on this point that Quine's theory must finally falter. In the first place, letting sign-designs endow meaning to themselves or their fellows won't achieve what Quine needs. If a sign-design has intrinsic sense, then to know the sign-design must be to know the sense. But then the expression would never need translation everyone who knew of it would know what it meant, and there wouldn't be anything to prevent it being directly used in any language. Further, the sentence's meaning could not be held indeterminate, for its sense is by hypothesis here self-determinate. Moreover, the sentence could not be said to condition another sentence - to constrain or promote its deployment - for then its conditioning role would be its significance, and once that was known, the role could be displaced onto any arbitrarily chosen symbol, which would violate the hypothesis of the sui generis significance of the sentence. The same error would be involved in holding that any sentence was its translation, however arbitrarily. Such sentences, therefore, cannot be held as things to which the meanings of other sentences are relative, nor can they be said to supplement the conditions of use of any expression, nor should they be taken as the cause of the underdetermination of meaning and truth by experience, if such there be.

In the second place, there aren't such things as intrinsically meaningful expressions, as we can tell from the fact that if presented with unfamiliar sign-designs, we can't form the slightest defensible intuitive notion of their sense. Even if we are presented with many such designs, and are allowed to know how they may be used relative to each other, at best all we can do is discern the logical relations they might be taken to exemplify, on assumption that they have a meaning at all (e.g., coassentibles are consistent). Alternatively, we might suppose that some of them are about others of them, if some seem conditionally assented to upon the physical display of others. But the first possibility is allowed for by Positivism, where logical relations are held to be materially empty. And in the second possibility, we merely have some expression qua sign-designs serving as the observable conditions of use for other expressions qua meaningful expressions, as when the latter remark the physical properties of the former - again, no intrinsic meaning. 
If it is true that data ultimately underdetermine theory, and that experience underdetermines meaning, we have at least seen that the residual slack cannot be taken up by anything internal to linguistic form. I suspect, however, that there is a way to provision empirical control for every expression in language holistically conceived.

\title{
BIBLIOGRAPHY
}

DUMMETT, Michael, 1978, "The Significance of Quine's Indeterminacy Thesis", in Truth and Other Enigmas, London: Gerard Duckworth \& Company Limited. QUINE, W. V. O., "Ontological Relativity", "Epistemology Naturalized", in Ontological Relativity and Other Essays, New York and London: Columbia University Press.

_, 1970, Philosophy of Logic, Engelwood Cliffs, N. J., Prentice Hall Inc., chapter 1.

1960, Word and Object, Cambridge, Massachusetts: The M. I. T. Press.

-, 1980, "Two Dogmas of Empiricism", in From a Logical Point of View, Cambridge, Massachusetts and London, England, Harvard University Press. SCHICK, Karl, Dec. 1972, "Indeterminacy of Translation", The Journal of Philosophy.

\section{ARE SPEAKING AND MEANING EVALUATIVE ACTIVITIES?}

\author{
Alexander Matthews, Salisbury, Zimbabwe
}

In this paper, I wish to suggest that speaking and communicated meaning are evaluative activities. "Evaluative" here means based on personal judgement as opposed to what is desirable. In other respects such evaluations are nothing like moral and aesthetic judgements. I use the term here in a provocative sense, and not as a comparison with ethics. Such comparisons and differentiations though interesting, must be dealt with elsewhere. In regard to conversation at least, I will suggest that speaking and meaning - what is conveyed - are not rule-governed forms of behaviour. There is a sense in which they are, but only if they are at one remove from conversation. The meaning can then be regarded as the sentence observed in writing as a result of contextual considerations, including what is actually said.

The Theoretical ineffability of meaning ${ }^{1}$ defies an historical account of the use of that term. The suggestion or prescription is that it is an impossible term to deal with. This may be so conceptually, if the historical use of the term is too confused to stand up to inspection. In what follows, in the first of its two characteristics "meaning" 